\title{
Pain from brain: Can we remodel neural circuitry that generates phantom limb pain and other forms of neuropathic pain?
}

\author{
Thomas Elbert* \\ University of Konstanz, Department of Psychology, Germany
}

From occasional stabbing to excruciating pain, as though the limb is set on fire: Nobody had informed Corporal P about phantom limb sensations when his right lower arm was amputated following a blast in Afghanistan. At first Mr. P was surprised that he could still feel his right fingers and hand for months after the amputation. But then he realized that the brain structures that are related to the feeling, movement and pain in his arm would still exist after amputation. Could it be that the neural substrate that had once controlled the perception and movement of his arm were now giving rise to phantom limb sensations? Over the course of the years following his amputation, the veteran started to feel as if his hand was moving higher up, as if it were directly connected to the stump. The phantom sensations of his forearm were no longer there and slowly, over time, he began to feel pain in his now-absent hand. At first the pain appeared only occasionally, but subsequently, the tingling and sharp pain that occurred at precise locations on his phantom hand became more frequent with each passing year. The pain, although now an "illusion" to him, was more devastating than any "real" pain, resulting from the stimulation of nociceptors. When he formed a fist with his phantom fingers, the flexion would not stop, until he would feel how his fingernails, slowly, penetrated the palm.

The report of Thomas Weiss, Caroline Dietrich, Wolfgang Miltner and colleagues from the University of Jena [1] in this issue, demonstrates how to use knowledge from behavioural and neuroscience to develop treatment for this clinical condition.

Somatosensory information is conveyed from the body's surface to the somatosensory cortex and from there, it is relayed to adjacent neural networks. Subsequent activity of so-called "hidden units" is determined by the activities of the representational input units and the weights on the connections between the input and the hidden units. Hence, hidden units construct their own representations of the input and thus loose the spatial homuncular ordering. Therefore, the organization of these layers is not accessible by current forms of brain imaging - interacting with stimulus and response representation, these layers represent space for self-organization and "freedom". The weights between the input and hidden units determine the extent to which hidden layers become active. Neural connectivity and thus information coding by these hidden layers

\footnotetext{
* Tel.: +49 753188 4609; fax: +497531884601

E-mail address: Thomas.Elbert@Uni-Konstanz.de
}

remain flexible throughout life. The behaviour of the output units and this may or may not include a signal for pain - depends on the activity of the hidden layers and the weights between the hidden and the output units. If the sensory input from a body part to its brain representation had been severed by damage, the respective area of the brain will not lie dormant but adapts to such interruptions of normal functioning by means of reorganization and plastic alterations. Evidence from animal and human studies suggest that amputation or disuse of a limb leads to an invasion of adjacent cortical zones into the one formerly representing the amputated limb [1-3,5-7].

At the same time, increased use of the remaining limb leads to an expansion of its cortical representation and with it to increased dexterity. By means of practice, for example, one can acquire a complex motor task, such as playing the violin, which involves quick movements of the fingers. In order to perform with the corresponding needs, the brain is able to alter its circuitry, and it does so with additional practice $[2,4]$. Thus, there is never any portion of the brain, which goes unused; there will be no "unused space". A brain region, disconnected from its original input, will not stay inactive for long. The nerve cells from the inactive part of the brain become rapidly involved in the activity that is going on in neighboring areas [2]. Correspondingly, we can observe two such changes to become evident in the brain of persons with an upper arm amputation:

(1) The area of the brain that is responsible for the intact arm is used more often and with increasing skillfulness must process more information than usual. Therefore this area becomes enlarged.

(2) The brain structures that had processed information from the now amputated arm should also show reorganization [4].

There are various relationships between these neuroplastic events and the subjective phantom phenomena [7]. More than half of cases like Corporal $P$ will report strong phantom limb pain, and in these the remodeling of the somatosensory brain areas is extreme. Areas that were originally responsible for a hand and fingers were seen to process information from the lip or face $[2,6,7]$. However, as mentioned above, we can properly image only the "input" and "output" layers of cortex, which mirror with their homuncular organization the arrangement of the receptors in the periphery - we do not yet know how to decode the information in the "hidden" layers of this gargantuan associative network housed in the cerebral 
cortex. We also have not yet studied the role of the cerebellum in these plastic alterations.

While continuous plastic reorganization of neural structures enables the central nervous system circuitry to conform to the current needs of an organism and thus usually would confer an obvious adaptive advantage, plasticity bears the risk for maladaptive reorganization. This is obviously the case for phantom-limb pain. Similar processes may result in other forms of neuropathic pain or itching. The varied susceptibility to developing post-therapeutic neuralgia after an attack of shingles, for instance, may be related to the reorganizational CNS processes triggered by one or several shingles episodes. Any alterations to the afferent input, be it at the level of the peripheral nerve, the dorsal root ganglion or dorsal root, or the central nervous system can thus lead to maladaptive pain.

Treatments of this conditions have included relaxation techniques, massage therapy to the residual limb, biofeedback, meditation, acupuncture, neuro-stimulators, physical therapy, behaviour therapy and counseling and the administration of various drugs. Despite isolated reports of success (for review see, e.g. [7]), no treatment has been beneficial on more than a temporary basis.

Since bottom-up mechanisms apparently underlie both the cortical disorder and the phantom-limb pain, it seems possible that a behavioural intervention could be of value in reducing or eliminating phantom pain. Dietrich and colleagues in this issue report testing whether they can reverse some of these reorganizational processes by motivating and training the patients to frequently use their stump and prothesis in a more systematic manner, particularly in behaviorally relevant tasks. According to the use-dependent changes indicated above, some of the plastic alterations may be erased and with it, the phantom pain may subside. The Jena researchers employed procedures derived in part from a therapy, termed constraint-induced $(\mathrm{CI})$ movement therapy, developed by Taub and co-workers that has been found to be effective in substantially improving the rehabilitation after CNS-injury [8]. Following this logic, Dietrich, Weisz and colleagues introduced the training of use of a prosthesis with somatosensory feedback. As opposed to the heuristic treatment attempts mentioned above, this innovative approach has been based on theoretical knowledge derived from basic research in behavioural psychology and the neurosciences.
As in CI movement therapy, the authors administered twoweeks of massed practice in the use of a hand prosthesis with somatosensory feedback on grip strength. The strength was detected by pressure sensors located in the bend between thumb and index finger of the prosthetic hand and fed back through eight electrodes to the skin of subject's stump. This procedure would reinstate a meaningful feedback of hand use to the somatosensory cortex in behaviorally relevant tasks, i.e., during prosthetic hand use. The outcome showed the expected increase in functionality and with it a decrease in phantom limb pain. While the authors did not monitor changes in cortical organization, previous research strongly would suggest that such an alteration has occurred [2]. These changes may be lasting until another behaviorally relevant task engages the plastic processes to remodel the cortical machinery yet another time.

The authors demonstrates feasibility of the procedure and show that treatment of phantom limb pain maybe a reachable goal, given that a two-week training is already sufficient to produce clinically relevant improvement. While the sample size was small and the study cannot dismantle what ultimately may have caused the observed changes, the report offers hope for those in pain.

\section{References}

1] C. Dietrich, K. Walter-Walsh, S. Preißler, O.W.Witte, G.O. Hofmann, W.H. Miltner T. Weiss, Sensory feedback prosthesis reduces phantom limb pain: proof of a principle, Neuroscience Letters (2011).

[2] T. Elbert, B. Rockstroh, Reorganization of human cerebral cortex: the range of changes following use and injury, The Neuroscientist 10 (2004) 129-141.

[3] T. Elbert, H. Flor, N. Birbaumer, S. Knecht, S. Hampson, E. Taub, Extensive reorganization of the somatosensory cortex in adult humans after nervous system injury, Neuroreport 5 (1994) 2593-2597.

[4] T. Elbert, C. Pantev, C. Wienbruch, B. Rockstroh, E. Taub, Increased cortical representation of the fingers of the left hand in string players, Science 270 (1995) 305-307.

[5] T. Elbert, A. Sterr, H. Flor, B. Rockstroh, S. Knecht, C. Pantev, C. Wienbruch, E. Taub, Input-increase and input-decrease types of cortical reorganization after upper extremity amputation in humans, Experimental Brain Research 117 (1997) 161-164.

[6] H. Flor, T. Elbert, C. Wienbruch, C. Pantev, S. Knecht, N. Birbaumer, W. Larbig, E. Taub, Phantom limb pain as a perceptual correlate of massive cortical reorganization in upper limb amputees, Nature 375 (1995) 482-484.

[7] H. Flor, L. Nikolajsen, T.S. Jensen, Phantom limb pain: a case of maladaptive CNS plasticity? Nature Reviews Neuroscience 7 (2006) 873-881.

[8] E. Taub, G. Urswatte, T. Elbert, New treatments in neurorehabilitation founded on basic research, Nature Reviews Neuroscience 3 (2002) 228-236. 